\title{
Chronic administration of EP4-selective agonist exacerbates albuminuria and fibrosis of the kidney in streptozotocin-induced diabetic mice through IL-6
}

\author{
Riyaz Mohamed, Calpurnia Jayakumar and Ganesan Ramesh
}

Diabetic nephropathy is currently the most common cause of end-stage renal disease in the western world. Exacerbated inflammation of the kidney is known to contribute acceleration of nephropathy. Despite increased COX-2-mediated production of prostanoid metabolite PGE2, knowledge on its involvement in the progression of diabetic kidney disease is not complete. Here, we show the cross talk of the PGE2-EP4 pathways and IL-6 in inducing albuminuria and fibrosis in an animal model of type 1 diabetes. Hyperglycemia causes enhanced COX-2 expression and PGE2 production. Administration of PGE2 receptor EP4-selective agonist ONO-AE1-329 for 12 weeks exacerbated fibrosis and albuminuria. Diabetes-induced expression of inflammatory cytokines TNF $\alpha$ and TGF $\beta 1$ was enhanced in EP4 agonist-treated mice kidney. In addition, urinary excretion of cytokines (TNF $\alpha$ and IL-6) and chemokines (MCP-1 and IP-10) were significantly more in EP4-treated mice than vehicle-treated diabetes. Diabetes-induced collagen I and CTGF expression were also significantly higher in EP4-treated mice. However, EP4 agonist did not alter macrophage infiltration but increased cytokine and chemokine production in RAW264.7 cells. Interestingly, EP4-induced IL-6 expression in the kidney was localized in proximal and distal tubular epithelial cells. To confirm further whether EP4 agonist increases fibrosis and albuminuria through an increase in IL-6 expression, IL-6-knockout mice were administered with EP4 agonist. IL-6-knockout mice were resistant to EP4-induced exacerbation of albuminuria and diabetes and EP4-induced fibrosis. Our data suggest that EP4 agonist through IL-6 induces glomerulosclerosis and interstitial fibrosis, and IL- 6 represents a new factor in the EP4 pathway.

Laboratory Investigation (2013) 93, 933-945; doi:10.1038/labinvest.2013.85; published online 1 July 2013

KEYWORDS: albuminuria; chronic kidney disease; EP4 agonist; inflammation; nephropathy; prostaglandin E2

Diabetic nephropathy is the most common cause of endstage renal disease requiring dialysis in the United States ${ }^{1,2}$ and worldwide. ${ }^{3}$ The incidence of diabetic nephropathy in this country has increased substantially over the past few years. Between 20 and $40 \%$ of patients with diabetes ultimately develop nephropathy. The natural history of diabetic nephropathy differs according to the type of diabetes and whether microalbuminuria (defined as $>30 \mathrm{mg}$ but $<300 \mathrm{mg}$ albumin in the urine per day) is present. If left untreated, $80 \%$ of people who have type 1 diabetes and microalbuminuria will progress to overt nephropathy (ie, proteinuria characterized by $>300 \mathrm{mg}$ albumin excreted daily), whereas only $20-40 \%$ of those with type 2 diabetes over a period of 15 years will progress.
Currently, there are no effective therapies available to prevent the development of diabetic nephropathy. Therefore, to develop innovative therapeutic concepts to prevent the development and progression of diabetic nephropathy, a comprehensive understanding of the pathophysiology of this disease is mandatory.

Arachidonic acid metabolism through cyclooxygenase (COX) enzyme generates several metabolites that are known to cause inflammation as well as suppression of inflammation. One such metabolite is prostaglandins that arise from the enzymatic metabolism of arachidonic acid by prostaglandin $\mathrm{G} 2 / \mathrm{H} 2$ synthase (COX) and further metabolism by specific synthases. There are two isoforms of COX: COX-1, the constitutive enzyme, and COX-2, the 
inducible enzyme. The functional outcome of these metabolites is cell type and context specific. For example, whole-body inhibition of COX causes kidney injury, whereas podocyte-specific deletion of COX-2 is protective against diabetic nephropathy. ${ }^{4}$ Moreover, inhibition of COX-1 and -2 before ischemia reperfusion suppressed development of CKD and fibrosis. ${ }^{5}$

Our recent study and studies from other groups have shown that PGE2 levels were highly elevated in urine of diabetic mice. ${ }^{6-8}$ The inducible form of COX-2 expression also is elevated in diabetic kidney. The pharmacological activity of PGE2 is carried out via four different cell-surface receptor subtypes: EP1, EP2, EP3, and EP4. Of these four receptors, three are involved in modulation of cyclic adenosine $5^{\prime}$-monophosphate (cAMP) levels (EP2 and EP4 increase cAMP levels, EP3 reduces cAMP levels). The fourth receptor, $\mathrm{EP} 1$, is involved in regulating intracellular calcium levels. All four of the EP receptors have been localized in the kidney and are responsible for distinct actions of $\mathrm{PGE}_{2}$. The $\mathrm{EP} 1, \mathrm{EP} 3$, and EP4 receptors are present in the collecting duct. EP4 has also been shown to be expressed in the glomerulus, and EP2 mRNA has been localized to the outer and inner medulla of the rat kidney. ${ }^{9,10}$

The functions of these receptors are also cell type and context dependent. For example, administration of EP4 agonist protects against tubular injury in acute and chronic model (partial nephrectomy). ${ }^{11}$ However, overexpression of EP4 in podocyte in the same chronic model exacerbated podocyte injury and albuminuria. Moreover, deletion in podocyte causes protection against podocyte injury and albuminuria. ${ }^{12}$ Several studies have shown that PGE2 has anti-inflammatory activities in vitro, but recent in vivo studies have showed the opposite effects in the model of experimental autoimmune encephalomyelitis (EAE) where PGE2-enhanced Th1 and Th17 pathways mediated inflammation. ${ }^{13}$

Therefore, present study was initiated to clarify the role of the PGE2-EP4 pathways and their interaction with cytokines using highly selective agonist in chronic kidney diseases due to type 1 diabetes. In this report, we show that ONO-AE1-329, a highly selective and potent functional EP4-receptor agonist, exacerbated inflammation and fibrosis in the kidney of streptozotocin (STZ)-induced diabetic mice through increasing IL-6 production.

\section{MATERIALS AND METHODS Induction of Diabetes}

The induction protocol was described by the Animal Models of Diabetic Complication Consortium (AMDCC). ${ }^{14}$ The AMDCC recommends caution when high-dose protocol is used to induce diabetes due to known direct cellular toxicity of STZ. The Institutional Animal Care and Use committee of the Georgia Health Sciences University approved all of the protocols and procedures using animals (approval number 2011-0348). Eight-week-old C57BL/6J mice or
IL-6-knockout mice (Jackson Laboratories) were given STZ (100 mg/kg BW in citrate buffer) as a single dose. One week after injection, blood glucose was measured to confirm diabetes. Renal function and body weight were monitored every 4 weeks by measuring serum creatinine. Twelve weeks after induction of diabetes, 24-h urine was collected, and animals were killed to collect kidney tissue and blood. Citrate buffer was administered to control animals, which were subjected to the same treatment as the diabetic animals. One group of diabetic mice received ONO-AE1$329(0.1 \mathrm{mg} / \mathrm{kg}$ BW/day, subcutaneously) and other group received vehicle. ONO-AE1-329 is a gift from ONO Pharmaceuticals, Japan, and is known to be a specific agonist for EP4 receptors. ${ }^{15,16}$

\section{Renal Function}

Renal function was assessed by measurements of serum creatinine (Cat. No. DZ072B; Diazyme Labs, USA).

\section{Cytokine and Chemokine Measurement}

Cytokines and chemokines in plasma and urine were measured using ELISA kit from eBioscience.

\section{Urine Albumin Quantification by ELISA}

Twenty-four-hour urine was collected using a metabolic cage. Urine volume was measured and centrifuged at 12000 r.p.m. for $10 \mathrm{~min}$. The supernatant was aliquoted and stored at $-80^{\circ} \mathrm{C}$ until used. Urine albumin was measured using ELISA kit (Cat. No. E90-134; Bethyl Laboratories, Montgomery, TX, USA).

\section{Quantification of mRNA by Real-Time RT-PCR}

Real-time RT-PCR was performed in an Applied Biosystems 7700 Sequence Detection System (Foster City, California, USA). Total RNA of $1.5 \mu \mathrm{g}$ was reverse transcribed in a reaction volume of $20 \mu \mathrm{l}$ using Omniscript RT kit and random primers. The product was diluted to a volume of $150 \mu \mathrm{l}$, and $5 \mu \mathrm{l}$ aliquots were used as templates for amplification using the SYBR Green PCR amplification reagent (Qiagen) and gene-specific primers. The primer sets used were: mouse TNF $\alpha$ (forward: $5^{\prime}$-GCATGATCCGCGACGTG GAA-3'; reverse: $5^{\prime}$-AGATCCATGCCGTTG GCCAG-3'), MCP-1 (forward: 5'-ATGCAGGTCCCTGTCATG-3'; reverse: 5'-GCTTGAGGTGGTTGTGGA-3'), ICAM-1 (forward: 5'-AGATCACATTCACGGTGCTG-3'; reverse: 5'-CTTCAGA GGCAGGAAACAGG-3'), COX-1 (forward: $5^{\prime}$-GAATGCCA CCTTCATCCGAGAAG-3'; reverse: 5'-GCTCACATTG GAGAAGGACTCC- $3^{\prime}$ ), COX-2 (forward: 5'-GCGACATAC TCAAGCAGGAGCA-3'; reverse: 5'-AGTGGTAACCGCTCA GGTGTTG- $3^{\prime}$ ), and IL-6 (forward: 5'-GATGCTACCAAAC TGGATATAATC-3'; reverse: 5'-GGTCCTTAGCCACTCCT TCTGTG-3'). The amount of DNA was normalized to the $\beta$-actin signal amplified in a separate reaction (forward primer: $5^{\prime}$-AGAGGGAAATCGTGCGTGAC-3'; reverse: 5'-CAATAGTGATGACCTGGCCGT-3'). 


\section{Cell Culture}

RAW264.7 cells (ATCC) were grown to confluence using advanced RPMI medium containing 5\% serum in a six-well plate. On the day of treatment, advanced RPMI medium without serum was added, and cells were treated with $100 \mathrm{nM}$ of EP4 agonist (ONO-AE1-329) or vehicle (saline). RNA was isolated $6 \mathrm{~h}$ after treatment for quantification of inflammatory gene expression.

Peritoneal macrophages were isolated by injecting mice with $3 \mathrm{ml}$ of RPMI medium, gently massaging the belly, and then aspirating the fluid. Cells were resuspended in RPMI medium 1640 containing 10\% FCS and plated at a cell density of $1-5 \times 10^{5}$ cells per well of a six-well plate. Twenty-four hour after plating, non-adherent cells were removed by washing, and fresh medium was replaced. On the day of treatment, advanced RPMI medium without serum was added, and cells were treated with $100 \mathrm{nM}$ of EP4 agonist (ONO-AE1-329) or vehicle (saline). RNA was isolated $6 \mathrm{~h}$ after treatment for quantification of inflammatory gene expression.

\section{Western Blot Analysis}

Protein extraction from kidney and western blot analysis was carried out as described previously. ${ }^{17,18}$ The membrane was probed with rabbit anti-fibronectin, $\alpha$-smooth muscle actin, collagen I, and collagen IV (Abcam, Cambridge, MA). Proteins were detected using enhanced chemiluminescencedetection reagents (Amersham Pharmacia Biotech). Protein loading was normalized to $\beta$-actin expression using anti-mouse actin antibody (Sigma-Aldrich, St Louis, MO).

\section{Histology and Immunostaining}

Kidney tissue was fixed in buffered $10 \%$ formalin for $12 \mathrm{~h}$ and then embedded in paraffin wax. For assessment of injury, $5 \mu \mathrm{M}$ sections were stained with periodic acid-Schiff (PAS) followed by hematoxylin. To quantify leukocyte infiltration, sections were stained with anti-mouse macrophage antibody (Abcam) (1:200 dilution) followed by goat anti-rat biotin conjugate. Color was developed after incubation with $\mathrm{ABC}$ reagent (Vector Lab). Stained sections were photographed, and five $\times 40$ fields of neutrophils were examined for quantification of leukocytes. To determine collagen I, collagen IV, and $\alpha$-smooth muscle actin expression, sections were stained with goat anticollagen I, collagen IV, and rabbit anti- $\alpha$-smooth muscle actin antibody (Abcam) (1:100 dilution) followed by rabbit anti-goat biotin conjugate. Color was developed after incubation with $\mathrm{ABC}$ reagent (Vector Lab). Stained sections were photographed using an Olympus inverted microscope with color CCD camera.

\section{Morphometry}

For each kidney, digital images of 15 randomly chosen consecutive glomeruli were captured with an Olympus DP72 color CCD camera (Olympus, Pittsburg, PA, USA) mounted on an Olympus light microscope by moving from cortex to the medulla in a serpentine fashion. The glomerular tuft was traced, and the enclosed area was calculated with Cellsens software (Olympus).

\section{Statistical Methods}

All assays were performed in duplicate. The data are reported as mean \pm s.e.m. Statistical significance was assessed by an unpaired, two-tailed Student's $t$-test for single comparison or ANOVA for multiple comparisons.

Table 1 Characteristics of STZ-induced type 1 diabetes and the effects of EP4 agonist on body weight, kidney weight, and blood glucose in WT mice

\begin{tabular}{lcccc}
\hline & $\begin{array}{c}\text { Non-diabetic } \\
\text { vehicle-treated } \\
\text { control }\end{array}$ & $\begin{array}{c}\text { Non-diabetic } \\
\text { EP4-treated } \\
\text { control }\end{array}$ & $\begin{array}{c}\text { Diabetic } \\
\text { vehicle-treated } \\
\text { mice }\end{array}$ & $\begin{array}{c}\text { Diabetic } \\
\text { EP4-treated } \\
\text { mice }\end{array}$ \\
\hline Glucose (mg/dl) & $217 \pm 11$ & $159 \pm 25$ & $682 \pm 35^{*}$ & $637 \pm 41^{*}$ \\
BW (g) & $28 \pm 0.6$ & $27 \pm 1$ & $20 \pm 1^{*}$ & $22 \pm 1^{*}$ \\
KW (mg) & $320 \pm 12$ & $307 \pm 6$ & $391 \pm 19^{*}$ & $362 \pm 20^{*}$ \\
KW/BW (mg/g) & $11 \pm 0.5$ & $11 \pm 0.5$ & $20 \pm 2^{*}$ & $17 \pm 1^{*}$ \\
Serum creatinine & $0.1 \pm 0.04$ & $0.1 \pm 0.01$ & $0.14 \pm 0.03$ & $0.2 \pm 0.03$ \\
Mortality (\%) & 0 & 0 & $34^{* *}$ & $20^{*}$
\end{tabular}

Abbreviations: BW, body weight; KW, kidney weight.

Data are mean \pm s.e.m. for $N=6-10$ per group.

${ }^{*} P<0.05$ vs vehicle and EP4-treated non-diabetic control.

${ }^{* *} P<0.001$ vs all other groups.

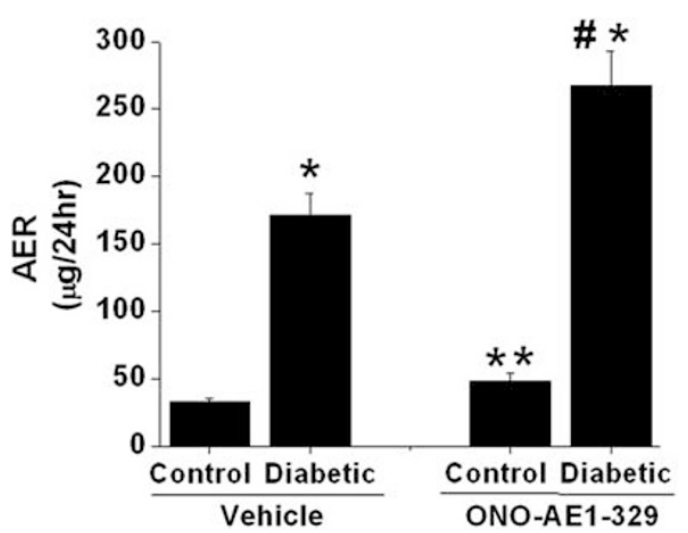

Figure 1 Albumin excretion rate (AER) in control, diabetic, and EP4 agonist-treated non-diabetic control and diabetic mice. Twenty-four-hour urine was collected, and urine albumin was measured by ELISA as described in Materials and Methods. Diabetes induced a significant rise in the excretion rate of albumin in urine over control mice, which was exacerbated in EP4 agonist-treated mice. ${ }^{*} P<0.001$ vs all other groups. $\# P<0.001$ vs diabetes vehicle-treated animals. ${ }^{* *} P<0.05$ vs vehicletreated control. $N=6-10$. 

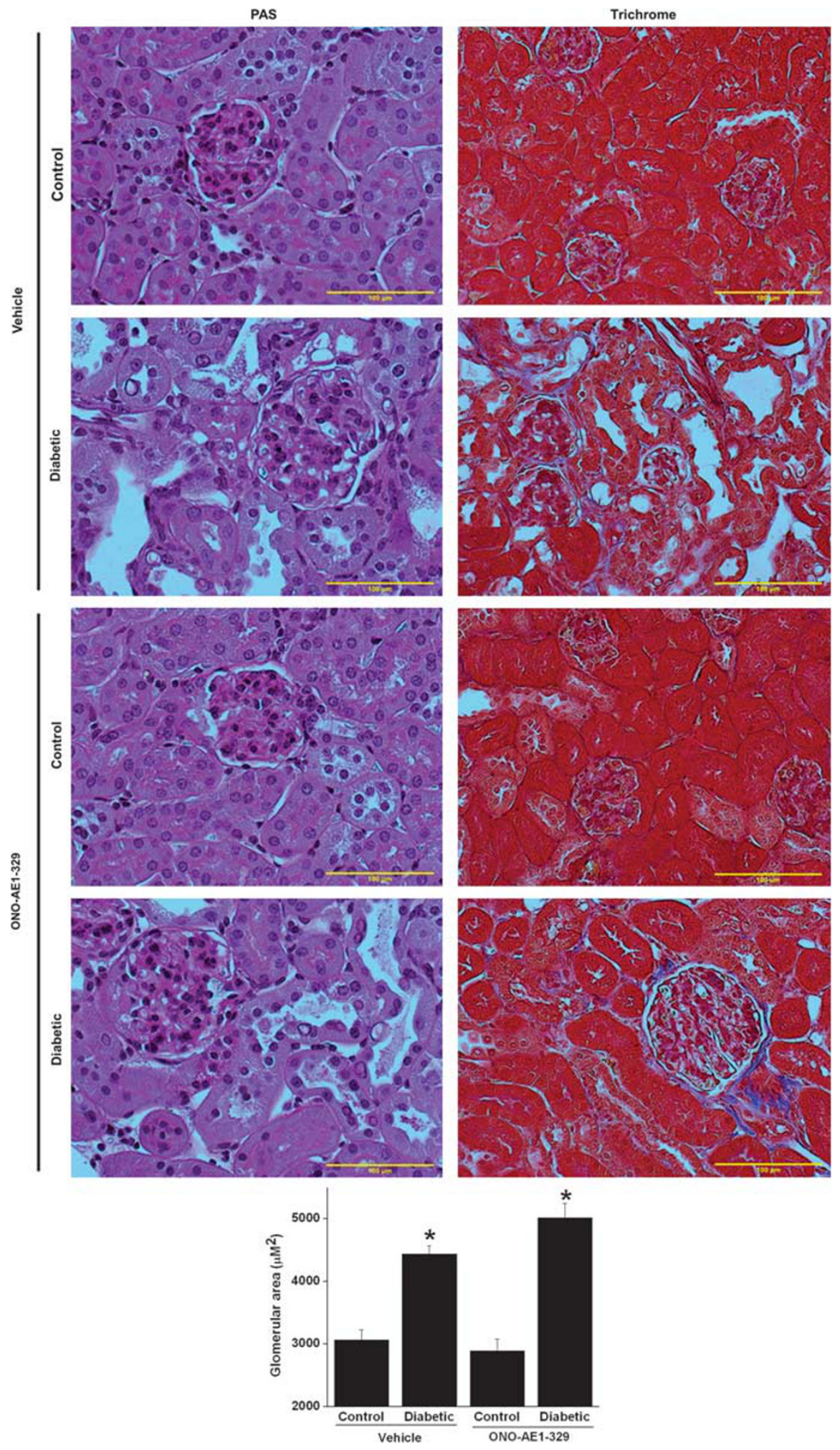

Figure 2 Diabetes and EP4 administration increased glomerular expansion and fibrosis. Top left panels: tubular damage and glomerular expansion were assessed in kidney cortex using PAS-stained tissue sections as described in Materials and Methods. Control non-diabetic kidneys from the vehicleand EP4 agonist-treated mice show normal tubular structure, but vehicle- and EP4-treated diabetic kidney shows high levels of tubular atrophy, apoptosis, brush border loss, and necrosis. Top right panels: EP4 agonist administration exacerbate kidney fibrosis determined by Masson's trichrome staining. Vehicle-treated and EP4 agonist-treated non-diabetic control do not have any significant Trichrome staining (blue). Diabetes induced increase in trichrome staining, which was increased further by administration of EP4 agonist. Bottom bar graphs: glomerular area quantification. Thirty to fifty glomeruli area was measured using (Cellsens Standard software) Olympus microscope with $\times 40$ objectives. ${ }^{*} P<0.001$ vs other groups. $N=6-8$. 

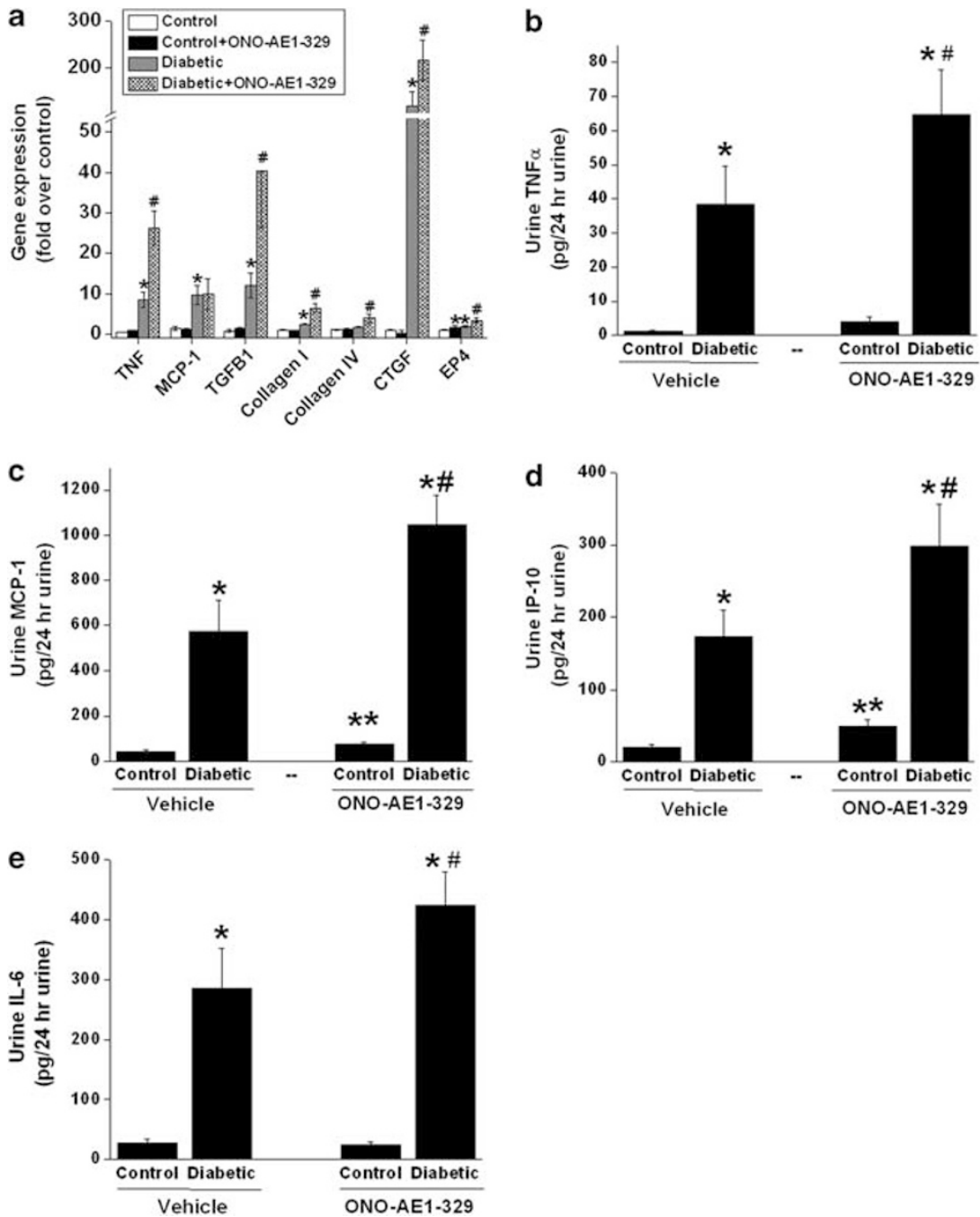

Figure 3 EP4 administration exacerbate diabetes-induced inflammation in kidney. (a) Kidney cytokine and chemokine gene expression was determined by real-time RT-PCR. Vehicle-treated diabetic mouse kidney showed a significant increase in expression of cytokine and chemokine genes, profibrotic genes, and extracellular matric genes, which were significantly enhanced in EP4 agonist-treated diabetic mice. ${ }^{*} P<0.001$ vs control. ${ }^{\#} P<0.05$ vs vehicle-treated diabetic mice. $N=4$. (b-e) Cytokine and chemokine excretion in 24-h urine was determined by ELISA. Excretion of several cytokines and chemokines in urine is increased in vehicle-treated diabetic mice as compared with control mice, which was exacerbated in EP4 agonist-treated mice. ${ }^{*} P<0.001$ vs all other groups. ${ }^{\#} P<0.05$ vs vehicle-treated diabetic mice. ${ }^{*} P<0.05$ vs vehicle-treated control mice. $N=4$.

\section{RESULTS}

\section{Effect of EP4 Agonist on Blood Glucose, Body Weight,} and Kidney Weight

Control mice gained weight as expected. However, there was no significant increase in body weight in either WT or EP4 agonist-treated diabetic mice (Table 1). Blood glucose levels were increased significantly in diabetic mice after STZ injection and continued to be elevated throughout the study period but were not elevated in buffer-treated control groups (Table 1). Serum creatinine was not changed in any of the groups. Diabetes increased mortality significantly as opposed to EP4 agonist-treated mice. However, diabetes-induced kidney hypertrophy was not altered with EP4 agonist treatment (Table 1).

\section{Effects of EP4 Agonist on Urinary Albumin Excretion}

As shown in Figure 1, the urinary albumin excretion (UAE) rate was $32 \pm 2 \mu \mathrm{g} / 24 \mathrm{~h}$ in the vehicle-treated control group and increased to $171 \pm 16 \mu \mathrm{g} / 24 \mathrm{~h}(P<0.001 v s$ control $)$ at week 12 in diabetic vehicle-treated group. Administration of EP4 agonist in non-diabetic control mice itself significantly increased UAE to $48 \pm 6 \mu \mathrm{g} / 24 \mathrm{~h} \quad(P<0.05 v s$ vehicle-treated control). The effect on EP4 agonist on UAE was exacerbated in diabetic mice $(267 \pm 25 \mu \mathrm{g} / 24 \mathrm{~h}, P<0.001$ $v s$ vehicle-treated diabetic).

\section{EP4 Agonist Increases Glomerular Expansion and Fibrosis}

PAS-stained section showed a large percentage of cortical tubular atrophy, necrosis, loss of brush border, and apoptotic 

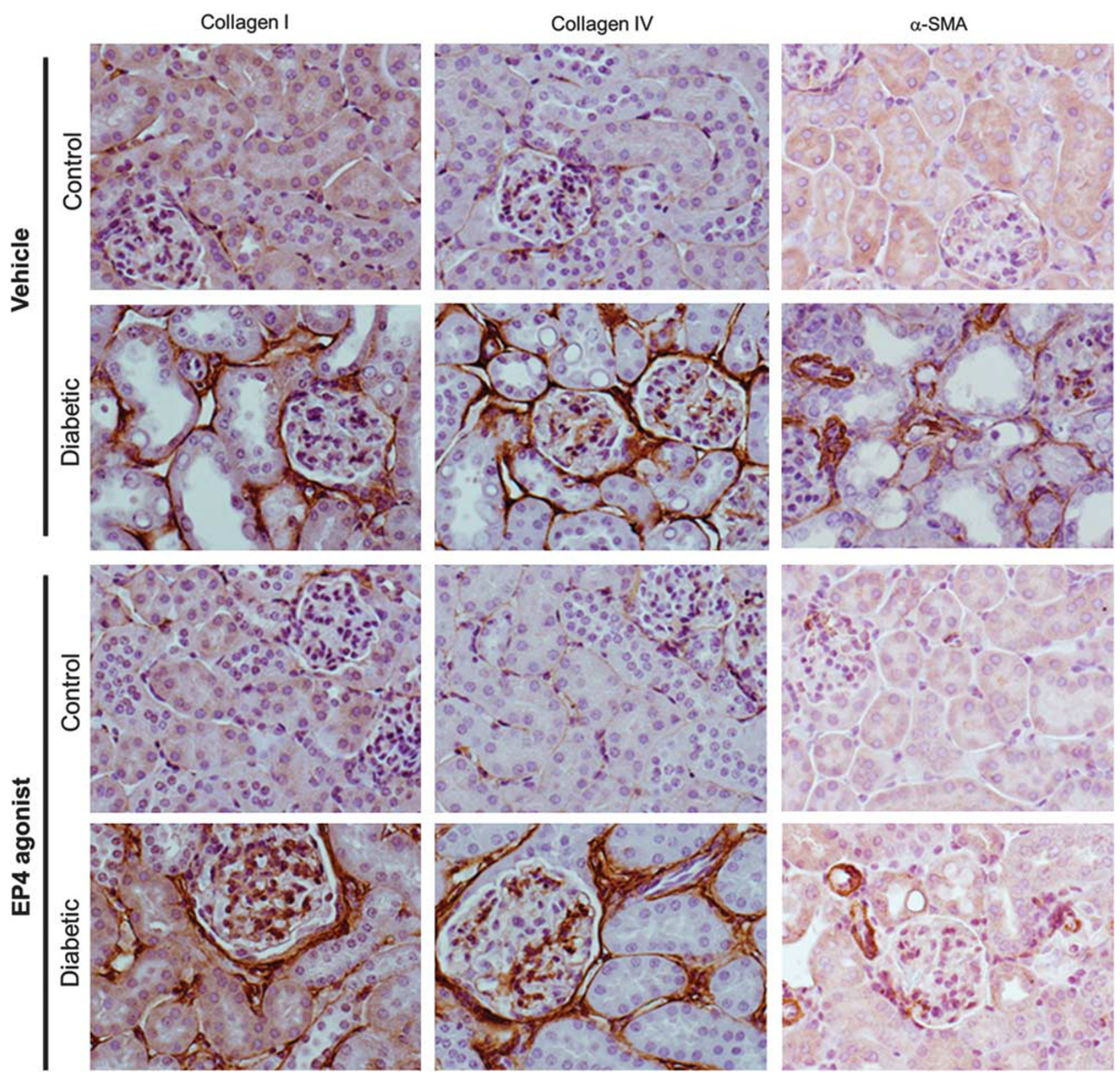

Figure 4 Diabetes induced fibrotic protein expression. Fibrotic protein expression was determined by immunostaining for collagen I, collagen IV, and $\alpha$-smooth muscle actin ( $\alpha$-SMA) in kidney tissue sections from control and diabetic animals with/without EP4 agonist treatment. Minimal or negligible staining was seen for collagen I, collagen IV, and $\alpha$-SMA in control mice treated with vehicle or EP4 agonist. However, staining was significantly increased in both vehicle-treated diabetic and EP4 agonist-treated diabetic kidney.

bodies in vehicle-treated diabetic mice kidney as compared with vehicle-treated control. Administration of EP4 agonist in non-diabetic control mice did not alter tubular morphology nor glomerular structure. However, administration of EP4 agonist in diabetic mice did not alter tubular injury but slightly increased diabetes-induced glomerular mesangial expansion (Figure 2).

Very low or negligible levels of staining were seen in nondiabetic control, vehicle-treated, and EP4-treated animal kidney, whereas a significant increase in Masson's trichrome staining was seen in vehicle diabetic kidney, which was increased even further in EP4 agonist-treated animal kidney (Figure 2).

\section{EP4 Agonist Regulate Inflammatory and Fibrotic Gene Expression}

As shown in Figure 3a, chronic diabetes increased the expression of cytokines (TNF $\alpha$ and TGF- $\beta 1$ ) and chemokines (MCP-1), profibrotic cytokines CTGF, and extracellular matrix protein gene (collagen I) in the kidney as compared with vehicle-treated control. Administration of EP4 agonist significantly enhanced the expression of both inflammatory 
a
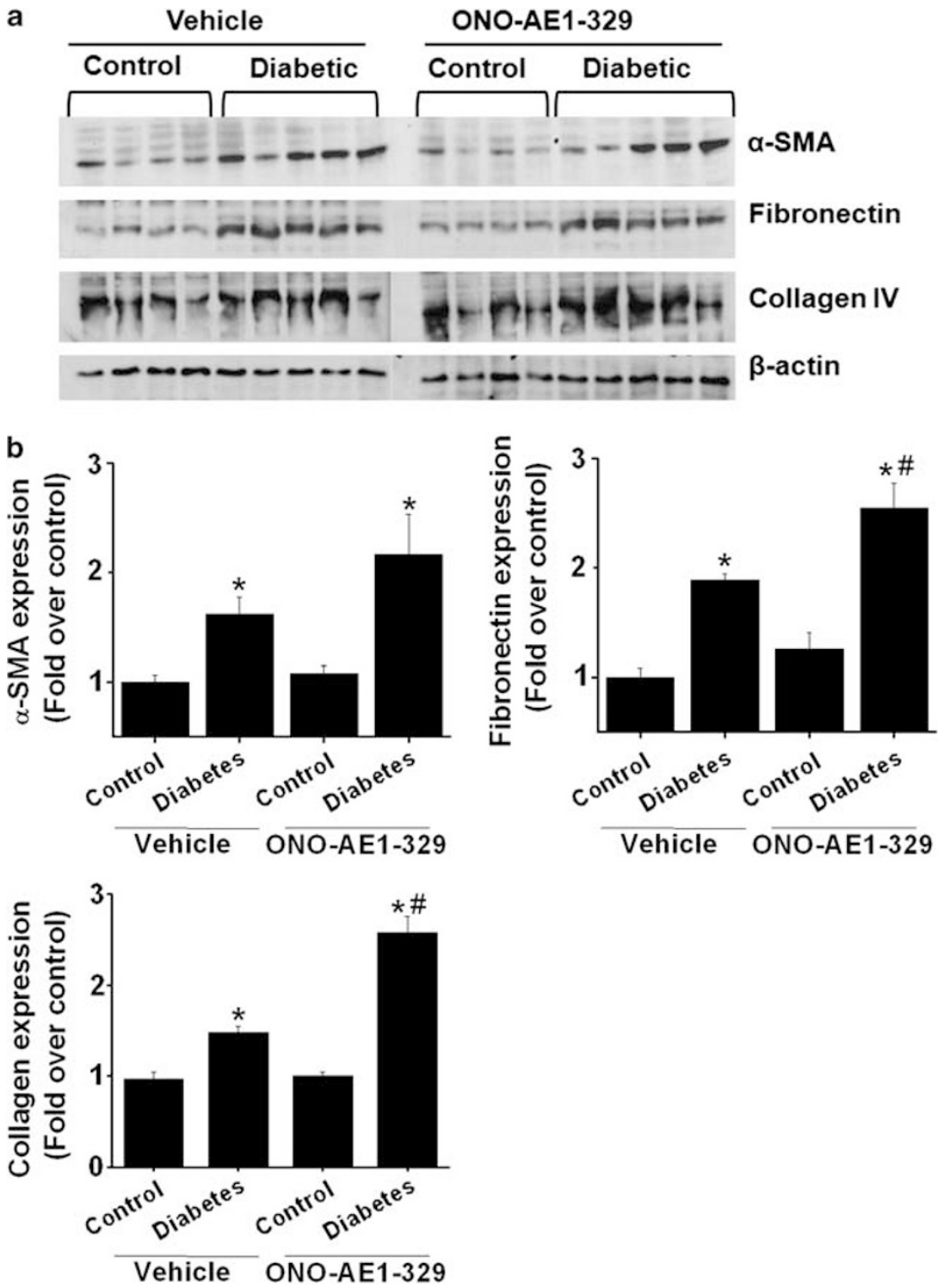

Figure 5 Quantification of extracellular matrix and fibrotic protein expression in kidney by western blot analysis (a). Diabetes significantly increased $\alpha$-SMA expression in diabetic kidney as compared with non-diabetic control, which was exacerbated by administration of EP4 agonist-treated diabetic mice (b). Densitometric quantification Western blot analysis for SMA, fibronectin and collagen shown in panel a. ${ }^{*} P<0.05$ vs all other groups. ${ }^{\#} P<0.05$ vs vehicle-treated diabetic mice. $N=4-5$.

genes as well as fibrotic genes in diabetic mice kidney. In addition, EP4 agonist administration also enhanced EP4-receptor expression.

Most of the cytokines and chemokines analyzed are undetectable or present in very low levels in the urine of control mice. Consistent with the increased expression of cytokines and chemokines in kidney, the excretion of cytokines and chemokines were also increased in urine of vehicle-treated diabetic mice as compared with vehicletreated control mice (Figures 3b-f). Administration of EP4 agonist alone in non-diabetic control mice significantly increased the chemokines MCP-1 and IP-10 excretion in urine. The excretion of inflammatory cytokines and chemokines were exacerbated with the administration of
EP4 agonist in diabetic mice as compared with vehicletreated diabetic mice.

\section{Effects of EP4 Agonist on Renal Fibrotic Protein Expression}

Consistent with Masson's trichrome staining, a large increase in immunostaining for both collagen I, collagen IV, and $\alpha$-SMA were seen in vehicle- and EP4 agonist-treated diabetic kidney (Figure 4). Consistent with Masson's trichrome and immunostaining, western blot analysis and quantification also showed a significant increase in fibrosis in vehicle-treated diabetic animal kidney, which was further increased in EP4 agonist-treated diabetic animal kidney (Figure 5). 

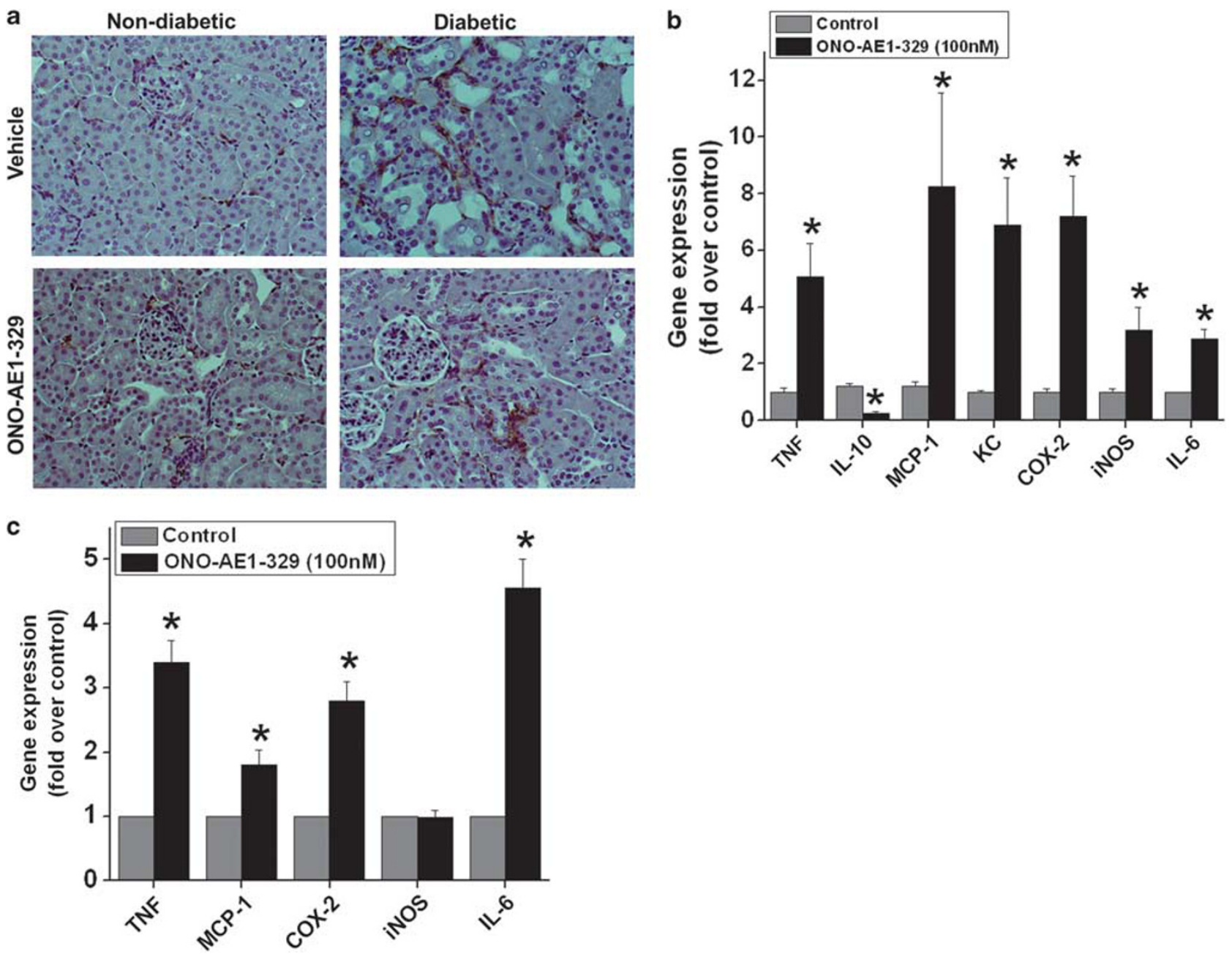

Figure 6 Macrophage infiltration into the kidney and inflammatory response with EP4 agonist treatment. (a) Macrophage infiltration into kidney was determined by immunostaining with anti-macrophage antibody as described in Materials and Methods. Vehicle- and EP4 agonist-treated control kidney showing no macrophage. Diabetic kidney showing increased macrophage infiltration. Administration of EP4 agonist did not alter diabetes-induced macrophage infiltration. RT-PCR analysis of inflammatory gene expression in RAW264.7 cells (b) and peritoneal macrophage (c) in response to EP4 agonist treatment. Treatment of EP4 agonist $(100 \mathrm{nM})$ for $6 \mathrm{~h}$ induced several inflammatory genes. ${ }^{*} P<0.001 \mathrm{vs}$ control. $N=4$.

\section{EP4 Agonist Does not Alter Diabetes-Induced Macrophage Infiltration into Kidney but Increased Inflammatory Cytokine Production}

Macrophage infiltration is known to be increased in diabetic kidney, and increased infiltration was associated with glomerular injury and interstitial fibrosis. To determine whether EP4 agonist enhanced macrophage infiltration, thereby increasing fibrosis over vehicle-treated diabetic kidney, immunolocalization of macrophage was carried out. As shown in Figure 6a, diabetes induced a large increase in macrophage infiltration into the interstitium, which was not changed in EP4 agonist-treated diabetic kidney.

Our recent studies have shown that PGE2 induced chemokine expression in proximal tubular epithelial cells. To determine, whether increased inflammatory cytokine and chemokine also come from infiltrating macrophage, RAW264.7 cells were treated with EP4 agonist, and expression of cytokines and chemokines was determined by PCR. Addition of EP4 agonist significantly increased inflammatory mediators TNF $\alpha$, IL-6, MCP-1, KC, COX-2, and iNOS but downregulated anti-inflammatory cytokine IL-10 (Figure 6b). Consistent with established macrophage cell line, freshly isolated peritoneal macrophage also showed a similar inflammatory response with EP4 agonist treatment (Figure 6c). Our results suggest that macrophages may contribute to the enhanced inflammatory response in EP4 agonist-treated kidney.

\section{EP4 Agonist-Mediated Increase in IL-6 Expression is Localized in Proximal and Distal Tubular Epithelial Cells as Well as Interstitial Cells}

To determine the cell type that produce IL-6 cytokines in response to diabetes and EP4 administration, IL-6 immunolocalization was performed. IL-6 expression is not 

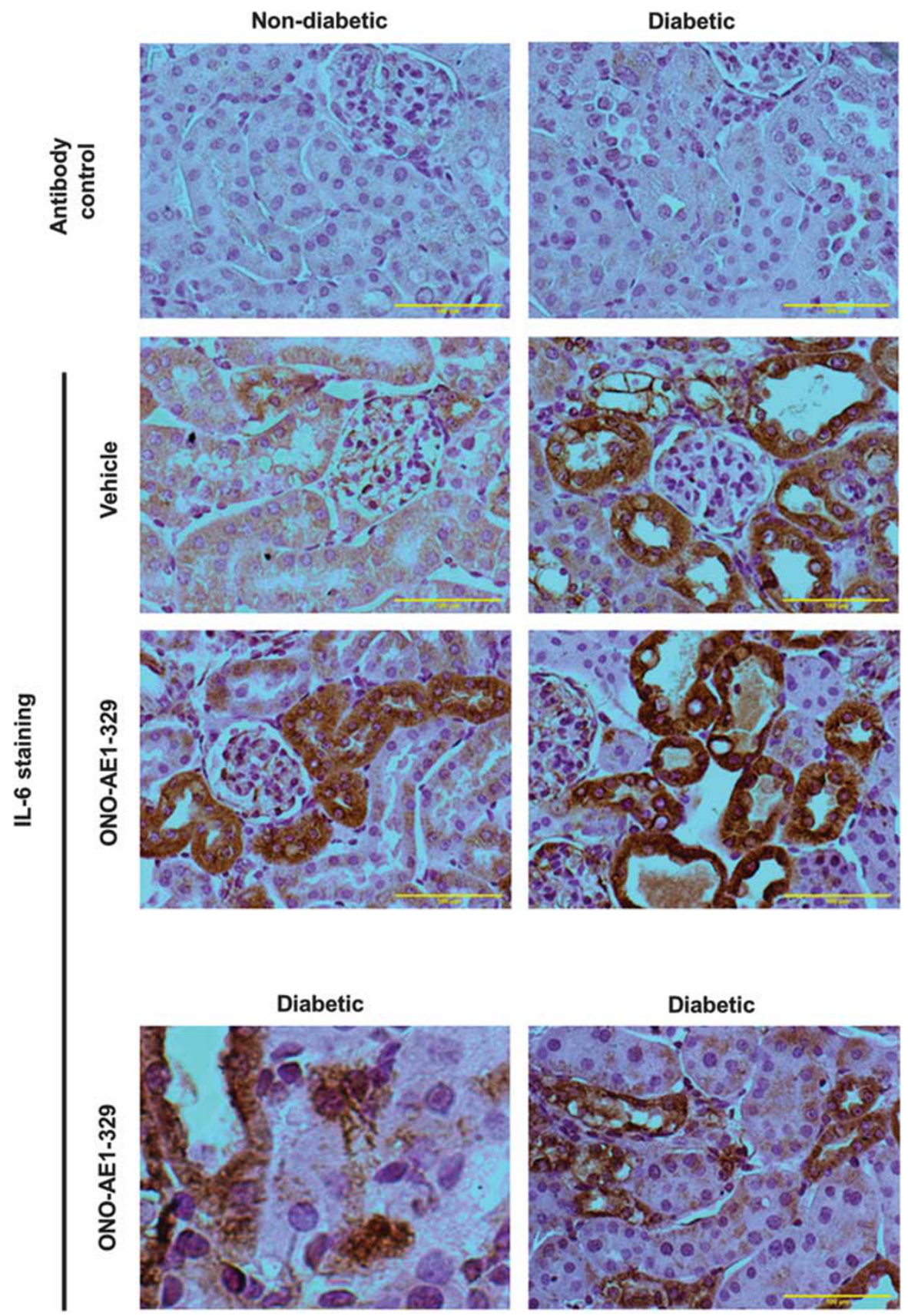

Figure 7 IL-6 immunolocalization in non-diabetic and diabetic mouse kidney treated with vehicle or EP4 agonist (ONO-AE1-329). No staining was seen with normal goat IgG primary antibody controls or vehicle-treated non-diabetic mouse kidney. Intense immune staining in proximal tubular epithelial cells was seen in diabetic mouse kidney. Ep4 agonist-treated non-diabetic mouse kidney showed increased levels IL-6 expression in proximal and distal tubular epithelial cells, which was further increased with diabetes. Some staining was also seen in interstitial cells of drug-treated diabetic mice.

detectable in vehicle-treated non-diabetic mouse kidney. However, administration of EP4 agonist in non-diabetic control animal increased IL-6 expression in tubular epithelial cells. Induction of diabetes also increased in IL-6 expression in the tubular epithelial cells, which was further increased in diabetic mice treated with EP4 agonist (Figure 7). Staining was also seen in interstitial cells and glomerulus but not as intense as seen in proximal tubular epithelial cells.

\section{IL-6-Knockout Mice are Resistant to EP4 Agonist- Mediated Exacerbation of Albuminuria and Fibrosis}

As shown in Figure 8 and Table 2, the urinary albumin excretion rate (AER) was increased significantly in diabetic wild-type (WT) and IL-6-knockout mice over control mice. Administration of EP4 agonist in non-diabetic IL-6-knockout mice did not increase the AER. However, administration of EP4 agonist increased AER significantly in WT diabetic mice but not in IL-6-knockout diabetic mice. 
To determine whether absence of exacerbation of AER in IL-6-knockout diabetic mice with EP4 agonist is associated with suppression of glomerular sclerosis and interstitial fibrosis, kidney sections were stained with Masson's trichrome. Very low or negligible levels of staining were seen in nondiabetic control vehicle-treated mice kidney. A significant increase in Masson's trichrome staining was seen in vehicletreated diabetic kidneys, which was increased even further in EP4 agonist-treated WT diabetic animal kidney that was suppressed in IL-6-knockout mouse kidney (Figure 9).

\section{DISCUSSION}

COX inhibition reduces albuminuria in clinical and experimental renal disease. COX-2 inhibition with SC58236

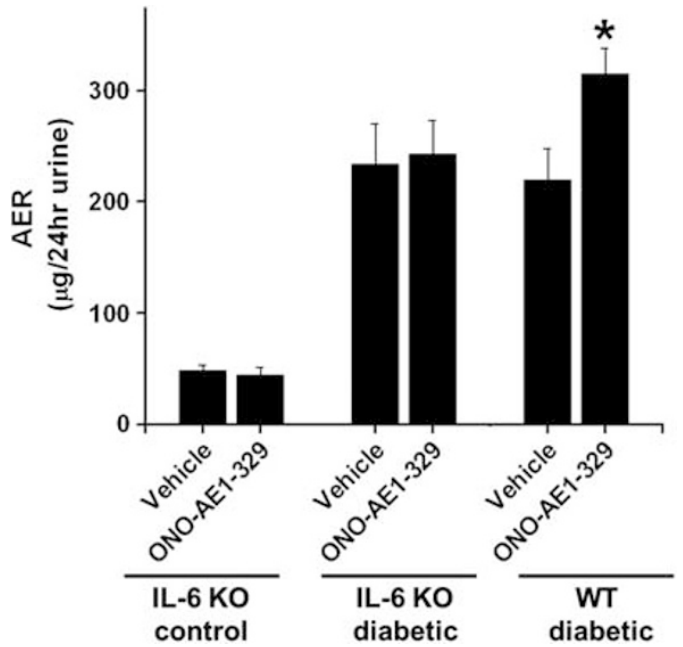

Figure 8 Albumin excretion rate (AER) in control, diabetic, and EP4-treated diabetic IL-6-knockout (IL-6 KO) and wild-type (WT) mice. Twenty-four-hour urine was collected and urine albumin was measured by ELISA as described in Materials and Methods. Diabetes induced a significant rise in the excretion rate of albumin in urine over control mice, which was exacerbated in EP4 agonist-treated WT mice but not IL-6 KO mice. ${ }^{*} P<0.001$ vs all other groups. $N=6-10$. blunted PGE2 synthesis and proteinuria while improving glomerulosclerosis in nephrectomized rats. ${ }^{19}$ Moreover, EP4 overexpression in podocytes exacerbated glomerular injury and albuminuria, whereas podocyte-selective deletion of EP4 resulted in protection against glomerulosclerosis and albuminuria in 5/6 Nx CKD model. ${ }^{12}$ Our results are consistent with this finding. Our result shows that chronic administration of EP4-selective agonist exacerbated inflammation, albuminuria, and fibrosis. The elevated COX-2 levels render the mice susceptible to glomerular filtration barrier injury after either adriamycin or puromycin aminonucleoside-induced nephrosis, whereas non-transgenic mice remain resistant. ${ }^{20,21}$ Moreover, podocyte-specific knockout of COX-2 in mice also protected kidney against diabetes-induced nephropathy and albuminuria. ${ }^{4}$ Our recent studies also showed that COX-2 is highly induced in proximal tubular epithelial cells after induction diabetes with STZ. Moreover, netrin-1 overexpression suppressed COX-2 expression and PGE2 production, which was associated with significant reduction in albuminuria and inflammation, ${ }^{6}$ suggesting an important role of COX-2 metabolites in diabetic nephropathy.

In EP4 agonist-treated diabetic kidney, tubulointerstitial fibrosis was significantly augmented compared with vehicletreated diabetic kidneys, indicating that the PGE2-EP4 pathway has an active role in the progression of tubulointerstitial fibrosis via EP4. Our results are consistent with earlier published report. ${ }^{12}$ Our recent studies and studies from other laboratories have shown that the renal expression level of COX-2 mRNA increased significantly after diabetes and obstructive nephropathy, $7,9,11,12,22$ suggesting that COX-2-derived prostanoids including PGE2 participate in the pathogenesis of tubulointerstitial fibrosis. In addition, we found that the expression level of EP4 mRNA increases after diabetes, which was further increased with EP4 administration. EP4 mRNA has been localized mainly in the glomeruli and the vascular wall in normal kidneys, ${ }^{23}$ but

Table 2 Characteristics of STZ-induced type 1 diabetes and the effects of EP4 agonist on body weight, kidney weight, and blood glucose in WT and IL-6 KO mice

\begin{tabular}{|c|c|c|c|c|c|c|}
\hline & $\begin{array}{l}\text { Vehicle-treated } \\
\text { IL-6 KO control }\end{array}$ & $\begin{array}{l}\text { EP4-treated IL-6 } \\
\text { KO control }\end{array}$ & $\begin{array}{c}\text { Diabetic } \\
\text { vehicle-treated } \\
\text { IL-6 KO mice }\end{array}$ & $\begin{array}{c}\text { Diabetic } \\
\text { EP4-treated IL-6 } \\
\text { KO mice }\end{array}$ & $\begin{array}{c}\text { Diabetic } \\
\text { vehicle-treated WT } \\
\text { mice }\end{array}$ & $\begin{array}{c}\text { Diabetic } \\
\text { EP4-treated WT } \\
\text { mice }\end{array}$ \\
\hline Glucose (mg/dl) & $119.75 \pm 11$ & $149 \pm 33$ & $524 \pm 53^{*}$ & $613 \pm 28^{*}$ & $603 \pm 25^{*}$ & $647 \pm 41^{*}$ \\
\hline BW (g) & $28 \pm 0.9$ & $29 \pm 1$ & $24 \pm 1^{*}$ & $24 \pm 1^{*}$ & $20 \pm 1^{*}$ & $22 \pm 1^{*}$ \\
\hline $\mathrm{KW}$ (mg) & $310 \pm 13$ & $317 \pm 4$ & $340 \pm 21^{*}$ & $378 \pm 11^{*}$ & $391 \pm 19^{*}$ & $362 \pm 20^{*}$ \\
\hline KW/BW (mg/g) & $11 \pm 0.7$ & $11 \pm 0.5$ & $14 \pm 1^{*}$ & $16 \pm 0.5^{*}$ & $20 \pm 2^{* *}$ & $17 \pm 1^{*}$ \\
\hline Serum creatinine & $0.1 \pm 0.04$ & $0.1 \pm 0.05$ & $0.13 \pm 0.04$ & $0.1 \pm 0.04$ & $0.14 \pm 0.03$ & $0.2 \pm 0.03$ \\
\hline Mortality (\%) & 0 & 0 & $25^{*}$ & $25^{*}$ & $35^{* *}$ & $10^{*}$ \\
\hline
\end{tabular}



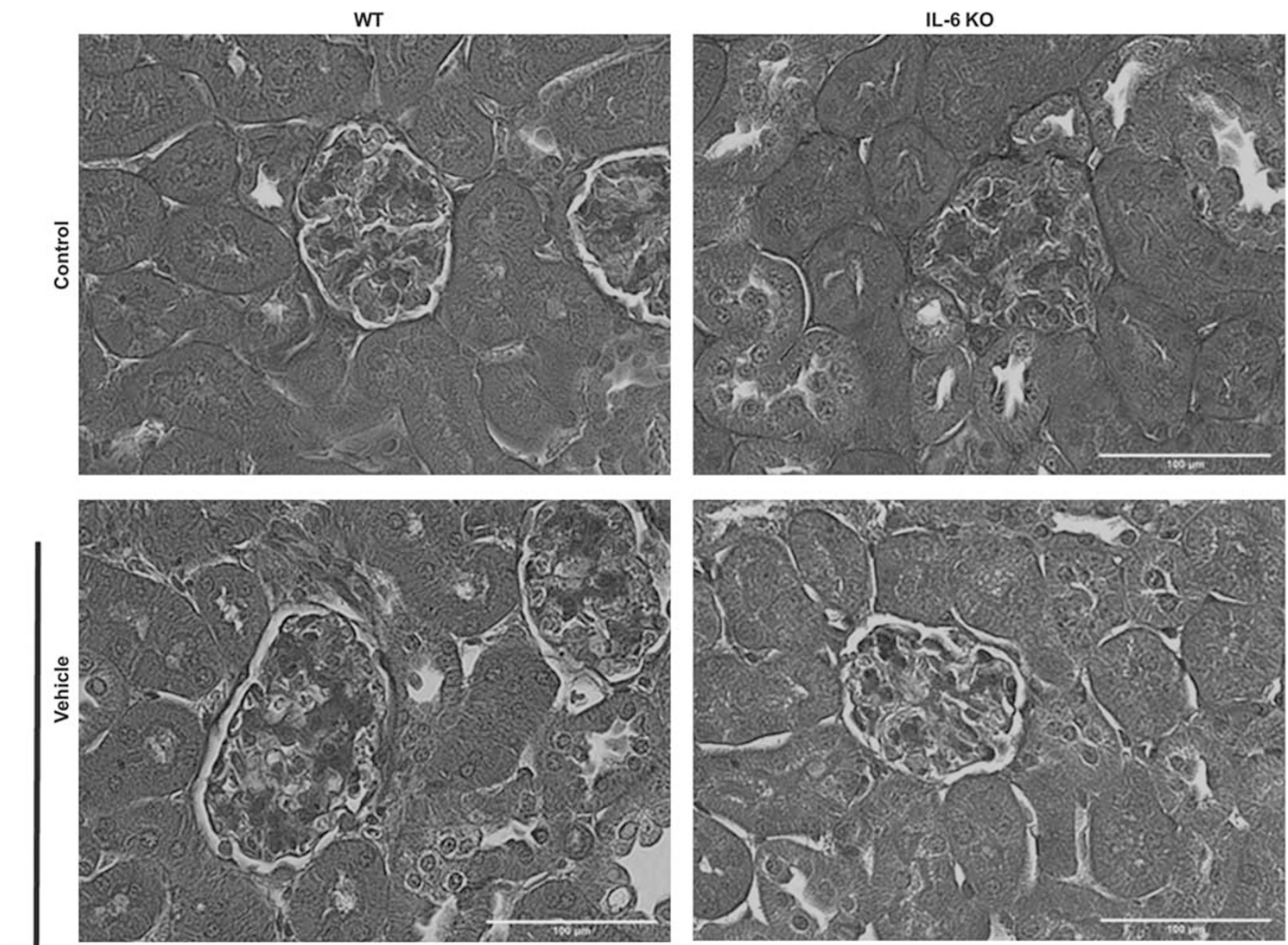

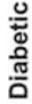
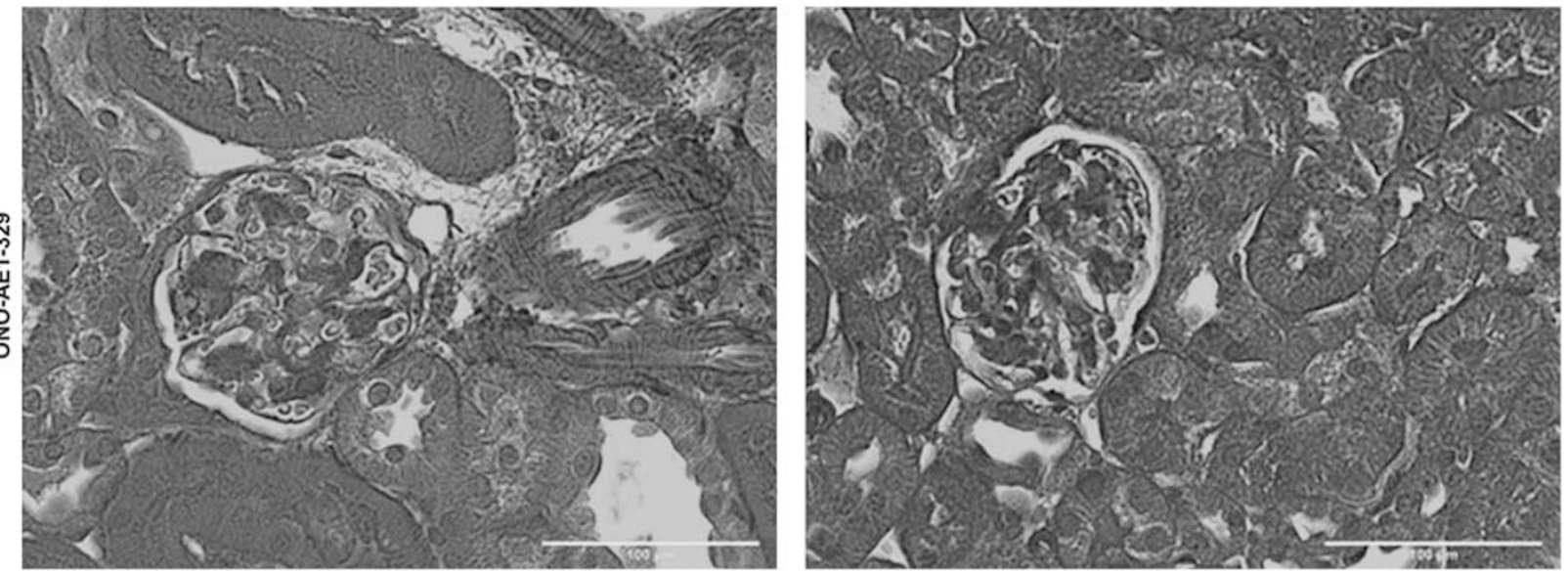

Figure 9 IL-6-knockout (IL-6 KO) mice are resistant to diabetes and EP4 agonist-induced exacerbation of glomerular sclerosis and interstitial fibrosis. Kidney fibrosis determined by Masson's trichrome staining. Vehicle-treated wild-type (WT) and IL-6 KO control mice does not have any significant trichrome staining (blue). Diabetes induced increase in trichrome staining of glomerulus and interstitium, which was increased further by administration EP4 agonist in WT but not in IL-6 KO mouse kidney.

highly induced in tubular epithelial cells, interstitial cells, and podocytes after diabetes and obstruction. ${ }^{12,22}$ Therefore, PGE2-EP4 system is present in infiltrating cells as well as parenchymal cells that may have a role in the development of interstitial fibrosis by acting on both tubular epithelial cells and interstitial cells. Consistent with this view, our results show that addition of EP4 agonist induced a large increase in cytokine and chemokine production in macrophage in vitro. Moreover, increased expression of IL-6 was localized in the proximal tubular epithelial cells in vivo. 
Administration of EP4 agonist exacerbated albuminuria in diabetic mice. Our observation is consistent with previous EP4 knockout and overexpression studies ${ }^{12}$ in podocytes. The mechanism through which EP4 agonist damage podocyte was not known. Increased perfusion pressure and hyperfiltration could be one of the mechanisms. This view was supported by previous findings that PGE2 mediated the glomerular hyperfiltration in early stages of diabetes. ${ }^{8,24}$ Consistent with this view, we also see a significant increase in urine volume in EP4-treated diabetic mice as compared with vehicle-treated diabetic mice.

The PGE2-EP4 system has been suggested to have proinflammatory ${ }^{25}$ and vascular actions. For example, the PGE2-EP4 signaling positively regulates the development of T-helper type 1 and type 17 subsets and determines the extent of immune inflammation. ${ }^{13}$ Consistent with this view, diabetes-induced inflammatory cytokine and chemokine production was exacerbated in EP4 agonist-treated mice. However, we do not know the source of these cytokines. Our in vitro studies with macrophages and proximal tubular epithelial cells ${ }^{6}$ showed that EP4 agonist can induce a large increase in inflammatory mediators in both macrophage and tubular epithelial cells. IL-6 expression is localized in tubular epithelial cells, suggesting that EP4 agonist-induced increase in IL-6 from epithelial cells may act on the macrophages in the interstitium and glomerulus to increase fibrotic and inflammatory response. We also show in our studies that EP4 agonist induces a profibrotic cytokine TGF- $\beta 1$, which is known to upregulate collagen and CTGF. Therefore, EP4 agonist may promote fibrosis in diabetes either directly or indirectly through TGF $\beta 1$.

Although a number of studies suggests that macrophages and their secreted components like IL-6 are known to mediate chronic kidney injury and fibrosis, opposing views have also been documented. ${ }^{26-32}$ To determine whether EP4 agonist exacerbate albuminuria and fibrosis through induction of IL-6, we administered EP4 agonist to IL-6-knockout mice. Interestingly, IL-6-knockout mice showed albuminuria similar to WT diabetic mice, but they are resistant to EP4 agonist-induced increase in albuminuria. In addition, IL-6-knockout mice showed a significant reduction in diabetes-induced fibrosis. These results suggest that IL-6 is downstream of EP4 signaling in mediating albuminuria.

In summary, our findings point to a cross talk between cytokines and PGE2-EP4 signaling in the development and progression of nephropathy. Activation of EP4 receptor contributes to the exacerbated albuminuria, inflammation, and fibrosis in a mouse model of type 1 diabetes and highlights the pleiotropic nature of the intrarenal actions of the prostanoid family.

\section{ACKNOWLEDGEMENTS}

This work was supported by a R01grant (1R01DK083379_01A3) to Ganesan Ramesh from NIH-NIDDK.

\section{DISCLOSURE/CONFLICT OF INTEREST}

The authors declare no conflict of interest.

1. Allan JC, Bertram K, Charles $\mathrm{H}$, et al. United States Renal Data System 2006 Annual Data Report. Am J Kidney Dis 2007;49:A6-A7.

2. Held PJ, Port FK, Webb RL, et al. The United States Renal Data System's 1991 Annual Data Report: an introduction. Am J Kidney Dis 1991; 18:1-16.

3. Marcussen N. A tubular glomeruli and the structural basis for chronic-renal-failure. Lab Invest 1992;66:265-284.

4. Cheng H, Fan X, Moeckel GW, et al. Podocyte COX-2 exacerbates diabetic nephropathy by increasing podocyte (pro)renin receptor expression. J Am Soc Nephrol 2011;22:1240-1251.

5. Feitoza CQ, Goncalves GM, Semedo P, et al. Inhibition of COX 1 and 2 prior to renal ischemia/reperfusion injury decreases the development of fibrosis. Mol Med 2008;14:724-730.

6. Mohamed R, Jayakumar C, Ranganathan PV, et al. Kidney proximal tubular epithelial-specific overexpression of Netrin-1 suppresses inflammation and albuminuria through suppression of COX-2mediated PGE2 production in streptozotocin-induced diabetic mice. Am J Pathol 2012;181:1991-2002.

7. Cheng HF, Wang CJ, Moeckel GW, et al. Cyclooxygenase-2 inhibitor blocks expression of mediators of renal injury in a model of diabetes and hypertension1. Kidney Int 2002;62:929-939.

8. Nasrallah R, Robertson SJ, Hebert RL. Chronic COX inhibition reduces diabetes-induced hyperfiltration, proteinuria, and renal pathological markers in 36-week B6-Ins2(Akita) mice. Am J Nephrol 2009;30: 346-353.

9. Jensen $B L$, Stubbe J, Hansen $P B$, et al. Localization of prostaglandin E2 EP2 and EP4 receptors in the rat kidney. Am J Physiol Renal Physiol 2001;280:F1001-F1009.

10. Breyer MD, Breyer RM. Prostaglandin E receptors and the kidney. Am J Physiol Renal Physiol 2000;279:F12-F23.

11. Vukicevic S, Simic P, Borovecki F, et al. Role of EP2 and EP4 receptorselective agonists of prostaglandin E2 in acute and chronic kidney failure. Kidney Int 2006;70:1099-1106.

12. Stitt-Cavanagh EM, Faour WH, Takami $\mathrm{K}$, et al. A maladaptive role for EP4 receptors in podocytes. J Am Soc Nephrol 2010;21: 1678-1690.

13. Yao C, Sakata D, Esaki Y, et al. Prostaglandin E2-EP4 signaling promotes immune inflammation through Th1 cell differentiation and Th17 cell expansion. Nat Med 2009;15:633-640.

14. Breyer MD, Böttinger $E$, Brosius $F C$, et al. for the AMDCC. Mouse models of diabetic nephropathy. J Am Soc Nephrol 2005;16: 27-45.

15. Esaki Y, Li Y, Sakata D, et al. Dual roles of PGE2-EP4 signaling in mouse experimental autoimmune encephalomyelitis. PNAS 2010;107: 12233-12238.

16. Liang $X$, Lin L, Woodling NS, et al. Signaling via the prostaglandin E2 receptor EP4 exerts neuronal and vascular protection in a mouse model of cerebral ischemia. J Clin Invest 2011;121:4362-4371.

17. Wang W, Brian RW, Ramesh G. Netrin-1 and kidney injury. I. Netrin-1 protects against ischemia-reperfusion injury of the kidney. Am J Physiol Renal Physiol 2008;294:F739-F747.

18. Ramesh G, Reeves WB. p38 MAP kinase inhibition ameliorates cisplatin nephrotoxicity in mice. Am J Physiol Renal Physiol 2005;289: F166-F174.

19. Wang JL, Cheng HF, Shappell S, et al. A selective cyclooxygenase-2 inhibitor decreases proteinuria and retards progressive renal injury in rats. Kidney Int 2000;57:2334-2342.

20. Cheng $\mathrm{H}$, Wang $\mathrm{S}$, Jo $\mathrm{Yl}$, et al. Overexpression of cyclooxygenase-2 predisposes to podocyte injury. J Am Soc Nephrol 2007;18:551-559.

21. Jo $\mathrm{Yl}$, Cheng $\mathrm{H}$, Wang $\mathrm{S}$, et al. Puromycin induces reversible proteinuric injury in transgenic mice expressing cyclooxygenase-2 in podocytes. Nephron Exp Nephrology 2007;107:e87-e94.

22. Nakagawa N, Yuhki Ki, Kawabe Ji, et al. The intrinsic prostaglandin E2-EP4 system of the renal tubular epithelium limits the development of tubulointerstitial fibrosis in mice. Kidney Int 2012;82:158-171.

23. Sugimoto $Y$, Namba T, Shigemoto R, et al. Distinct cellular localization of mRNAs for three subtypes of prostaglandin E receptor in kidney. Am J Physiol Renal Physiol 1994;266:F823-F828. 
24. Yao B, Xu J, Oi Z, et al. Role of renal cortical cyclooxygenase-2 expression in hyperfiltration in rats with high-protein intake. Am J Physiol Renal Physiol 2006:291:F368-F374.

25. Sakata D, Yao C, Narumiya S. Prostaglandin E2, an immunoactivator. J Pharmacol Sci 2010;112:1-5.

26. Chow F, Ozols E, Nikolic-Paterson DJ, et al. Macrophages in mouse type 2 diabetic nephropathy: correlation with diabetic state and progressive renal injury. Kidney Int 2004;65:116-128.

27. Furuichi $\mathrm{K}$, Wada $\mathrm{T}$, Iwata $\mathrm{Y}$, et al. CCR2 signaling contributes to ischemia-reperfusion injury in kidney. J Am Soc Nephrol 2003;14: 2503-2515.
28. Galkina E, Ley K. Leukocyte recruitment and vascular injury in diabetic nephropathy. J Am Soc Nephrol 2006;17:368-377.

29. Wang Y, Harris DCH. Macrophages in renal disease. J Am Soc Nephrol 2011;22:21-27.

30. Kielar ML, John R, Bennett M, et al. Maladaptive role of IL-6 in ischemic acute renal failure. J Am Soc Nephrol 2005;16:3315-3325.

31. Navarro-Gonzalez JF, Mora-Fernandez C. The role of inflammatory cytokines in diabetic nephropathy. J Am Soc Nephrol 2008;19:433-442.

32. Harcourt BE, Forbes JM, Matthews VB. Obesity-induced renal impairment is exacerbated in interleukin-6-knockout mice. Nephrology 2012;17:257-262. 